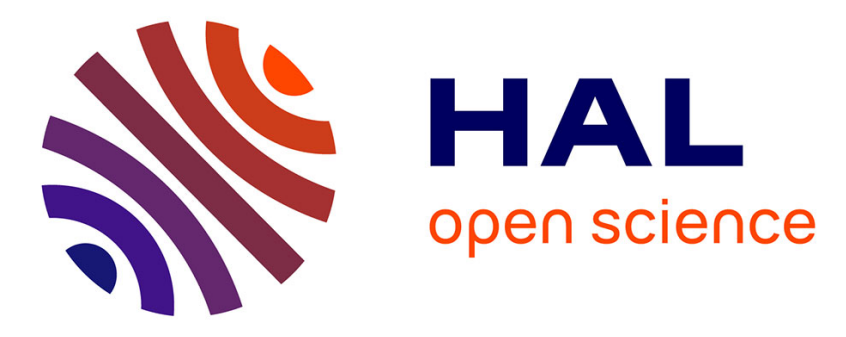

\title{
Globalisation, régulation et transformations des entreprises
}

Jean-Philippe Robé, Patrick Verley, Gabriel Galvez-Behar, Philippe Lefebvre

\section{To cite this version:}

Jean-Philippe Robé, Patrick Verley, Gabriel Galvez-Behar, Philippe Lefebvre. Globalisation, régulation et transformations des entreprises. Entreprises et Histoire, 2019, nº4 (1), pp.186-197. 10.3917/eh.094.0186 . hal-02444063

HAL Id: hal-02444063

https://hal-mines-paristech.archives-ouvertes.fr/hal-02444063

Submitted on 17 Jan 2020

HAL is a multi-disciplinary open access archive for the deposit and dissemination of scientific research documents, whether they are published or not. The documents may come from teaching and research institutions in France or abroad, or from public or private research centers.
L'archive ouverte pluridisciplinaire HAL, est destinée au dépôt et à la diffusion de documents scientifiques de niveau recherche, publiés ou non, émanant des établissements d'enseignement et de recherche français ou étrangers, des laboratoires publics ou privés. 


\section{GLOBALISATION, RÉGULATION ET TRANSFORMATIONS DES ENTREPRISES}

Avec

Jean-Philippe ROBÉ

Avocat international

et

Patrick VERLEY

Professeur émérite d'histoire économique

Université de Genève

Propos recueillis par

Gabriel GALVEZ-BEHAR

Professeur d'histoire contemporaine

Université de Lille

et

Philippe LEFEBVRE

Enseignant-Chercheur en Sciences de gestion

CGS, Mines ParisTech - PSL Research University

Débat à paraître dans Entreprises et Histoire, 2019/1, n94

Numéro spécial sur «Globalisation et transformation des entreprises »

Patrick Verley, auteur de L'échelle du monde. Essai sur l'industrialisation de l'Occident, $2^{e}$ éd., Paris, Gallimard, 2013 et Jean-Philippe Robé, auteur de Le temps du monde de l'entreprise. Globalisation et mutation du système juridique, Paris, Dalloz, 2015 répondent à nos questions sur la généalogie de la globalisation, l'évolution des entreprises en son sein et sur les risques actuels d'une bataille généralisée sans règles. 
Les termes de mondialisation et de globalisation sont souvent utilisés comme des motsvalises. Quelles représentations ou définitions de la mondialisation et de la globalisation ont votre préférence?

Jean-Philippe Robé : Je ne trouve pas cette question de vocabulaire très enrichissante. C'est un des seuls points de désaccord que j'ai avec Alain Supiot, qui insiste sur la différence à faire entre les deux termes ${ }^{1}$. Pourquoi pas. Mais comment pourrions-nous avoir un tel débat en anglais ? Il n'y a qu'un seul terme en anglais, globalization, et nous allons avoir du mal à en restreindre l'usage du fait d'un supplément de richesse de la langue française. Maintenant, sur le fond, je crois qu'il faut bien distinguer deux phénomènes - la géographie des échanges et les structures de pouvoirs - pour ensuite examiner leurs relations. La géographie des échanges s'appuie sur des pouvoirs qui permettent aux échanges d'exister; et les pouvoirs qui aident aux échanges influent sur leur géographie. Les premiers échanges au long cours ont été des échanges au sein d'empires qui précèdent les États comme structures de pouvoir englobantes. Il suffit alors de protéger quelques routes, de collecter quelques impôts sur les échanges commerciaux à quelques étapes incontournables, et l'alliance entre les marchands et les empires est faite. Mais on ne touche alors que la surface, une proportion infime des échanges, ceux du luxe. La globalisation, c'est l'extension progressive à l'ensemble de la population de l'accès à des biens produits un peu partout sur la planète. Fernand Braudel l'a rappelé toute sa vie : c'est quand la ville est entrée en jeu que le capitalisme est monté en puissance. Et, avec lui, de nouvelles formes d'alliance dans le système de pouvoir. Deux types d'alliance vont alors se lier, au croisement d'une multitude de facteurs. Le premier, la puissance des villes elles-mêmes. Les villes italiennes n'ont pas besoin d'État : elles sont des États, des puissances urbaines dominant leur campagne et n'ont besoin de personne. Les secondes, les villes allemandes, sauront jouer l'empire contre les pouvoirs locaux, et se liguer en hanses et guildes, capables de lever des armées et de protéger leurs échanges. Les troisièmes, telles les villes françaises, sont bien moins puissantes et font face à un État qui se développe tôt. Et elles ont besoin tôt de protection contre les pouvoirs féodaux locaux, abusifs, imprévisibles, prompts à les taxer mais pas à leur fournir les routes, le droit et la sécurité dont elles ont besoin pour les échanges. Elles vont très vite comprendre tout l'avantage qu'il y a à se positionner dans la hiérarchie féodale immédiatement comme vassales du roi. Et le roi, pas toujours mais souvent, aura l'intelligence de laisser les villes commercer pour pouvoir mieux les taxer. Hendrik Spruyt a écrit des pages éclairantes sur ces jeux d'alliances ${ }^{2}$.

La globalisation, c'est déjà cela : c'est l'apprentissage des jeux d'alliances entre les acteurs des jeux de l'échange ${ }^{3}$ et ceux des institutions politiques en construction. C'est ce développement parallèle de l'échange économique et de la construction du politique et, bien

\footnotetext{
${ }^{1}$ A. Supiot (dir.), Mondialisation ou globalisation ? Les leçons de Simone Weil, Paris, Collège de France, 2019.

${ }^{2}$ H. Spruyt, The Sovereign State and Its Competitors. An Analysis of Systems Change, Princeton, Princeton University Press, 1994,

${ }^{3} \mathrm{~F}$. Braudel, Civilisation matérielle, économie et capitalisme, $X V^{e}-X V I I I^{e}$ siècle, t. II : Les jeux de l'échange, Paris, A. Colin, 1979. 
plus tard, de l'élargissement du marché et de l'installation en profondeur de l'État. Avant sa remise en question par la phase de globalisation que nous vivons.

Patrick Verley : Pour ma part, je trouve qu'il n'est pas inutile de mettre un peu d'ordre dans la terminologie en se référant à l'étymologie et à l'histoire de l'usage de ces mots : internationalisation, mondialisation, globalisation. Internationalisation signifie développement des relations économiques entre les différentes nations ou plus exactement entre acteurs appartenant à des nations différentes. Logiquement ce terme n'aurait pas de sens tant qu'il n'existe pas de fait national, ce que les historiens considèrent en général comme un fait tardif, lié à la construction des États-nations en Europe à l'époque moderne. Pourtant le commerce entre ensembles politiques ou ethniques différents a toujours été l'objet d'une réglementation et d'une attention spécifiques de la part des gouvernants, qui visaient à l'empêcher ou au contraire à le favoriser (Co-Hong chinois (à Canton), compagnies à charte européennes). Les politiques de développement industriel impliquent également une régulation des échanges extérieurs. Louis XI a implanté l'industrie de la soie à Lyon afin de soulager le déficit de la balance commerciale du pays. Les $\mathrm{XVI}^{\mathrm{e}}$ et $\mathrm{XVII}^{\mathrm{e}}$ siècles ont été caractérisés en Europe par la conjonction entre la puissance économique et la puissance politique, jusqu'alors plutôt dissociées.

L'internationalisation enfin n'implique en soi ni une baisse des barrières aux flux de marchandises, de capitaux, d'hommes, de technologies ni une uniformisation des comportements des acteurs ni une convergence vers une similarité des structures des économies nationales. L'une des phases historiques où l'internationalisation a le plus vite progressé a été la fin du $\mathrm{XIX}^{\mathrm{e}}$ siècle et les premières années du $\mathrm{XX}^{\mathrm{c}}$ siècle, pourtant marquées par une montée des protectionnismes douaniers et un affrontement des nationalismes.

Le terme «mondialisation», assez récent dans la terminologie, devrait logiquement marquer un approfondissement et non un simple élargissement du concept précédent. En revanche le besoin que certains auteurs ont ressenti, après la Seconde Guerre mondiale, de créer le néologisme «mondialisation» révèle le désir de se distinguer des outils sémantiques existants. Si l'on est attentif à la gradation entre internationalisation et mondialisation, ce dernier terme, sauf à être un doublon inutile, devrait impliquer l'effacement des barrières administratives et l'uniformisation des règles, des pratiques et des comportements des acteurs économiques pour lesquels le critère de nationalité ne deviendrait plus discriminant. Néanmoins beaucoup d'auteurs n'y perçoivent qu'une généralisation des transactions ou des échanges (le phénomène international existe, à la limite, dès qu'il y a relation bilatérale) - économiques bien sûr mais également à d'autres niveaux, sans y voir un processus de création d'un espace original. 


\section{Le problème de l'imprécision de la notion de mondialisation se pose aussi d'un point de vue historique. En fait, il y a toujours eu des échanges au long cours. Comment peut-on distinguer les diverses vagues de mondialisation ? De votre point de vue, quand s'installe celle que nous traversons actuellement?}

Patrick Verley : À mon avis, l'idée de mondialisation implique deux critères, l'existence d'un système économique d'interdépendance au niveau des différents continents connus et qu'il soit à une échelle suffisante pour que des changements conjoncturels ou de politique économique dans l'un soient ressentis dans les autres à une échelle macroéconomique. On ajoutera deux autres points : l'existence ou non d'un leader qui impose ses changements aux autres et la conscience que les acteurs ont eue de cette interdépendance.

Ainsi la formation d'un système quadri-continental date incontestablement des $\mathrm{XVI}^{\mathrm{e}}$, $\mathrm{XVII}^{\mathrm{e}}$ et $\mathrm{XVIII}^{\mathrm{e}}$ siècles. La première marchandise dont le marché est mondial est le métal précieux, marchandise et monnaie, dont la principale source d'approvisionnement vient des mines américaines, transite par l'Europe, puis le Moyen-Orient et aboutit en Extrême-Orient. Le premier marché d'un grand produit identique à l'échelle du monde est celui des indiennes, suscité par le besoin des compagnies à charte d'équilibrer leurs paiements pour éviter d'avoir à équilibrer la balance Europe-Asie par du métal argent. L'activité industrielle européenne dépend largement du marché mondial de la monnaie-marchandise, de la conjoncture asiatique des indiennes d'Inde et de la demande des marchés américains eux-mêmes, dépendants du marché du sucre.

Jean-Philippe Robé : Ces problèmes de périodisation sont passionnants. Mais, selon moi, ne voir dans la globalisation actuelle que la deuxième étape d'un processus qui aurait débuté à partir de la fin du XIX ${ }^{\mathrm{e}}$ siècle, c'est se couper de ce qui permet de comprendre ce qui nous arrive. Il se passe quelque chose d'essentiel dans le dernier tiers du XIX siècle : c'est l'introduction dans un droit libéral individualiste de la société par actions qui lui est totalement contradictoire. Nous en vivons aujourd'hui encore les conséquences; et peut-être que cela va encore s'aggraver. Mais l'origine de la globalisation est bien plus lointaine et elle est inscrite à la fois dans ce que Braudel appelait le monde matériel et dans celui que j'appelle le monde des idées. Car je crois à la force des idées, particulièrement quand elles en viennent à être institutionnalisées. Et, selon moi, comprendre la globalisation impose d'en comprendre la généalogie à partir du renouveau urbain, au plan matériel, et de la révolution grégorienne, au plan des idées, et donc au $\mathrm{XI}^{\mathrm{e}}$ siècle. Je m'en expliquerai plus en détail dans le deuxième volume d'un diptyque intitulé Globalisation, le premier volume ayant pour sous-titre Le Système Monde de Pouvoirs et le second Généalogie de la globalisation. En Europe Grégoire VII pour la Chrétienté fonde l'ordre juridique englobant que va ensuite muter en Système des États puis en Système de Pouvoirs global. Le couple Philippe Le Bel - Guillaume de Nogaret (et plus généralement les rois architectes de l'État aidés des légistes) va donner vie au triangle « pouvoir, droit et effet de vérité » mis en avant par Michel Foucault dans la montée du pouvoir royal (voir son cours au Collège de France du 14 janvier 1976), qui a ensuite pu sortir victorieux de la lutte entre l'Empereur et le Pape.

Se met en place, à partir de ces évolutions majeures, un Système de Pouvoirs d'un type nouveau qui va conduire à une première métamorphose - celle de la naissance du Système des 
États que l'on peut faire naître avec les traités de Westphalie de 1648 qui mettent fin aux guerres de religion. Car si la première phase de la globalisation identifiée par Braudel laisse cohabiter plusieurs économies-monde, c'est bien l'économie-monde européenne qui va l'emporter sur les autres. Sa «supériorité » est une supériorité institutionnelle. C'est en Europe que s'invente l'État moderne qui va ensuite coloniser le monde et faire que le Système des États va occuper l'ensemble de la planète. Et il a survécu à la décolonisation, au moins à la surface des choses. Dans cette perspective, la seconde métamorphose est celle que nous sommes en train de vivre, et qu'il nous faut analyser de l'intérieur, ce qui est une chance extraordinaire mais aussi quelque chose de très périlleux. Nous vivons la fin du Système des États (ce qui ne veut absolument pas dire que les États vont disparaître) et, peut-être, la naissance d'un Système Monde de Pouvoirs, fait d'États, d'organisations internationales, d'entreprises multinationales, de marchés, locaux, régionaux, globaux dont il nous faut, enfin, comprendre le fonctionnement d'une manière débarrassée des idéologies.

Patrick Verley : Les facteurs politiques jouent bien évidemment mais il ne faut pas oublier les aspects proprement économiques. Avant le $\mathrm{XVI}^{\mathrm{e}}$ siècle et au $\mathrm{XVI}^{\mathrm{e}}$, deux facteurs sont fondamentaux : la pénurie monétaire impliquant une recherche permanente d'or (il y a des mines d'argent en Europe) et une balance du commerce des métaux précieux constamment déficitaire dans le commerce avec l'Asie. Au XVI ${ }^{\mathrm{e}}$ siècle s'ajoute un besoin d'extension de terres qui ne parviennent plus à nourrir la population dès qu'elle se met à croître, d'où l'extraversion vers l'ouest et l'est. Aux XVII ${ }^{\mathrm{e}}$ et XVIII' ${ }^{\mathrm{e}}$ siècles, le facteur de la monnaie et de l'équilibre des paiements, avec le déficit structurel de l'Europe sur l'Asie, reste prépondérant. Le développement de l'exportation d'argent d'Amérique centrale par les Espagnols vers Manille permet d'acheter les produits asiatiques qui vont déstructurer l'industrie mexicaine. Arrivées après les Hollandais qui ont monopolisé le commerce des épices, les compagnies française et anglaise font face à des difficultés financières et doivent développer le marché des indiennes pour se financer. Ce marché international des indiennes se développe en symbiose avec la traite esclavagiste et la demande sucrière européenne.

Enfin, au XIX ${ }^{\mathrm{e}}$ siècle, un fait passé trop souvent sous silence et essentiel est le retournement de la hiérarchie économique dans les échanges mondiaux. L'Asie exporte auparavant des produits manufacturés de haute technologie que les Européens vont peiner à copier avec le mouvement de transfert technologique du XVIII ${ }^{\mathrm{e}}$ siècle (les porcelaines, les colorants textiles...). En dehors de l'horlogerie, l'Europe exporte quant à elle des produits primaires. Entre 1800 et 1820, l'Asie devient exportatrice primaire (coton brut, indigo, thé...) et l'Europe vend des produits manufacturés à l'échelle mondiale. En outre, pour se créer des marchés, les Britanniques qui ont une balance des paiements courants très déficitaire prêtent donc des capitaux à l'étranger et font d'une pierre plusieurs coups. Ils prêtent aux indépendantistes latino-américains pour qu'ils se libèrent de la colonisation ibérique, trouvant dans le développement des emprunts d'État de ces pays des occasions de placement, et en même temps financent des marchés pour leur production industrielle entre 1820 et 1840 . Ces transferts de capitaux financent aussi la production de matières premières dont leur industrie et l'alimentation de leur population ont besoin. 
Quels sont les facteurs de causalité importants de chacune des différentes vagues de mondialisation ? À l'aune de ces facteurs, quelles sont les spécificités du phénomène actuel de mondialisation? Pour l'actuelle mondialisation, comment voyez-vous la hiérarchie ou poids relatif des facteurs à l'œuvre?

Jean-Philippe Robé : Je pense que le point de départ incontournable, c'est une compréhension du système de pouvoirs, du fait que dans toute société un peu sophistiquée, on trouve toujours le fonctionnement combiné de micro- et de macro-pouvoirs, les uns influençant les autres. Et des changements, endogènes ou exogènes, peuvent venir apporter des modifications qui peuvent radicalement affecter l'équilibre des pouvoirs existant à un moment donné dans un Système de Pouvoirs donné. Par exemple, le climat s'adoucit au $\mathrm{XI}^{\mathrm{e}}$ siècle. Changement exogène. Les rendements agricoles augmentent. Une main-d'œuvre se libère qui va rejoindre les villes; et commence ainsi une dynamique qui, peut-être encore aujourd'hui, a ses répercussions, même si elles sont évidemment très lointaines. Après, je ne suis pas sûr qu'il soit utile d'identifier des facteurs de causalité, ou que l'on puisse toujours le faire. Il y a plutôt des logiques de système, avec des causalités croisées, enchevêtrées. Par exemple, le développement des échanges de marché a besoin de l'État pour se développer : il lui faut des routes, des ponts, des canaux, du droit, des tribunaux, des huissiers, des poids et mesures, une langue, etc.

Dans la généalogie de la globalisation, on va donc passer de la Chrétienté au Système des États avec à la fois des dates charnières, précises, marquant des mouvements quasitectoniques dans l'évolution institutionnelle. Ces derniers ne doivent cependant pas empêcher de voir l'évolution de longue durée et l'institutionnalisation d'une relation d'échange constituante entre les acteurs du marché et leurs besoins, d'un côté, et l'État avec les siens de l'autre, qui aboutissent à la constitutionnalisation de l'État comme ordre libéral, protégeant les droits de propriété et les contrats, en échange d'un impôt acceptable puisque rémunérant notamment l'infrastructure et l'institutionnalisation du marché. La deuxième mutation que nous vivons est celle entraînée par la globalisation des entreprises qui met à mal les États en restreignant leurs moyens de remplir leurs trois fonctions : fournir l'infrastructure (les services publics), internaliser les externalités négatives et corriger les inégalités. La situation est aujourd'hui explosive à ces trois niveaux.

Patrick Verley : Un aspect me paraît ne pas devoir être oublié : la conscience du phénomène de mondialisation par les acteurs. Par exemple, à l'époque moderne, les tisserands bretons ou mexicains ne savent pas que leur sort est dépendant des marchés textiles mondiaux. Cette idée pose trois questions. La première est celle de savoir quoi dire sur l'avant $\mathrm{XVI}^{\mathrm{e}}$ siècle, c'est-à-dire à une époque où le système international ne comprend que trois continents, centré sur l'Océan indien et la mer de Chine qui inclut la côte africaine orientale, le Moyen-Orient et le bassin méditerranéen, le reste de l'Europe étant aux confins et intégré seulement par le marché de la monnaie, de l'or africain. Ensuite l'idée de mondialisation vient de la conscience que les populations en ont, celle que leur sort est lié à ce qui se passe très loin et sur lequel ils n'ont pas prise, ce qui explique son succès fin $\mathrm{XX}^{\mathrm{e}}$ siècle. Enfin l'histoire est toujours européocentrée, c'est-à-dire qu'elle parle de mondialisation quand l'ancien cœur européen est concerné 
par ce qui se passe ailleurs et non lorsque le cœur européen ou atlantique du système infligeait sa conjoncture ou sa politique ailleurs.

Jean-Philippe Robé : Il y a un point que je souhaiterais souligner. Je crois que, d'une manière générale, on néglige trop le rôle du droit. Ce n'était certainement pas le cas de Braudel ; un peu plus celui de Wallerstein ${ }^{4}$. J'admets que ce puisse être une déformation personnelle, mais je ne le crois pas. La première métamorphose marque l'avènement au niveau " macro » du concept de souveraineté, dans sa double déclinaison interne (au sein de l'État) et externe (entre États) et, au niveau «micro», de l'institutionnalisation de l'individu via ses droits fondamentaux, et singulièrement le droit de propriété, les droits d'autonomie dont l'autonomie contractuelle. La société libérale se voit comme une société auto-construite par les individus via le jeu de leur autonomie contractuelle et de leur autonomie décisionnelle en tant que propriétaires. L'échange économique international existe, mais il ne met guère en œuvre une production organisée au plan international, si ce n'est pour l'extraction des matières premières. Les échanges sont avant tout des échanges de marché.

A chaque étape, il faudrait exposer les instruments juridiques de l'extension du marché, et les instruments juridiques de la structuration de l'organisation, publique ou privée. Dans la première phase, il n'y a pas vraiment d'entreprise. Il y a des marchands qui s'organisent entre eux pour se protéger, avoir des règles communes, échanger avec un minimum de sécurité. C'est l'ère de la lex mercatoria, du droit de la ville aussi, qui « rend libre ». C'est le droit des foires, des paix. La frange mince du capitalisme, ce «troisième étage », qui a forcément à faire avec le pouvoir et son financement, c'est celui de grandes familles, telles que les Fugger, les Bardi, les Peruzzi. La famille, hier comme aujourd'hui, est un formidable instrument pour les activités s'appuyant sur la confiance, les activités fiduciaires. Ensuite, ce sont les instruments de la construction des marchés et de la production, l'État mercantiliste, constructiviste, jouant un rôle essentiel. Ce n'est qu'une fois l'État bien installé que l'on va séparer les rôles, rêver de marché autorégulateur. Mais l'extension des marchés va conduire à une extension de la société par actions et à une re-concentration du pouvoir via les droits de propriété dans les grandes entreprises, marqueur de la troisième phase.

\section{Les entreprises n'ont-elles cependant pas joué un rôle ?}

Jean-Philippe Robé : C'est dans la phase de passage du Système des États au Système Monde de Pouvoirs que les entreprises jouent un rôle fondamental. Ce sont elles qui sont à l'origine d'une recomposition du Système de Pouvoirs centré autour du Système des États en un Système de Pouvoirs global dans lequel elles ont des ailes alors que les États ont des racines, comme le disait Ulrich Beck ${ }^{5}$.

Précisément, la seconde métamorphose - celle que nous vivons - trouve sa source dans l'internationalisation des entreprises grâce à la concentration des droits de propriété sur les

\footnotetext{
${ }^{4}$ Voir notamment I. Wallerstein, Comprendre le monde. Introduction à l'analyse des systèmes-monde, Paris, La Découverte, 2009.

${ }^{5}$ U. Beck, Pouvoir et contre-pouvoir à l'heure de la mondialisation, Paris, Flammarion, 2005. 
actifs productifs au sein d'entreprises qui, du fait de leur existence en tant qu'organisations internationales privées, sans aucune existence juridique en tant que telles, vont pouvoir jouer les États entre eux. L'entreprise mondiale ayant comme support un groupe de sociétés implantées dans pratiquement tous les pays du monde, les échanges en son sein apparaissent comme des échanges de marchés, alors que ce sont des échanges organisés. Les échanges entre les diverses filiales apparaissent comme des ventes, avec des prix payés, alors que ce sont des ordres avec des rémunérations optimisées du point de vue de l'entreprise globale. On peut ainsi aisément déplacer la constatation comptable des profits réellement réalisés par l'entreprise au sein d'une filiale localisée dans un paradis fiscal. Ou alors on localise le travail là où il est le moins protégé, et ainsi de suite. La globalisation, parce qu'elle est une globalisation des échanges organisés, permet aux entreprises de réaliser un immense travail d'externalisation de leurs coûts. Elles peuvent ainsi accroître leurs profits sans créer de valeur, voire en détruisant de la valeur, comme on peut le constater avec le réchauffement climatique, qui est la plus grande externalité négative de tous les temps ${ }^{6}$. Clairement, l'absence d'une compréhension de l'entreprise par les économistes néo-classiques, qui ramènent tout à de l'échange de marché en ignorant les cadres institutionnels de l'échange, joue un rôle fondamental dans les désastres sociaux et environnementaux que nous vivons. Ils ramènent à des échanges de marché des échanges organisés qui privent les États de leurs moyens d'existence et donc des moyens de remplir leurs fonctions.

\section{Quels sont les principaux problèmes auxquels les mondialisations successives et l'actuelle mondialisation ont confronté les entreprises ? À quelles transformations des activités- opérations et des savoirs opérationnels dans les entreprises chacune des mondialisations conduit-elle?}

Jean-Philippe Robé : L'entreprise moderne ne naît vraiment qu'au XIX ${ }^{\mathrm{e}}$ siècle. Bien sûr, la révolution industrielle joue son rôle. Mais n'oublions pas la révolution juridique que constitue le droit de créer sans contrainte des sociétés par actions. Le développement des chemins de fer a joué un rôle fondamental. Leur construction appelle des concentrations importantes de capitaux et donc le recours à ce «merveilleux instrument du capitalisme » qu'est la société par actions ${ }^{7}$. L'extension du marché que permet le chemin de fer appelle l'industrialisation de la production, et donc d'autres concentrations de capital. Puis c'est le développement de la banque, de l'assurance, etc. Progressivement, toute activité ayant un peu d'ampleur va être structurée grâce à la société par actions. Puis ce sont les groupes de sociétés qui vont servir de support aux plus grandes entreprises, à l'échelle mondiale aujourd'hui. Ce développement des échanges organisationnels - interne aux entreprises - va appeler les progrès dans les transports, les communications, le traitement de l'information et être rendu possible grâce à eux. Des techniques juridiques aussi. Et des techniques de gestion, bien sûr, de plus en plus professionnalisées. Pour ces organisations, qui vont développer en interne les savoirs nécessaires à leur contrôle opérationnel, la globalisation a été surtout une source extraordinaire

\footnotetext{
${ }^{6}$ Voir notamment F. Aggeri et M. Cartel (dir.), Entreprises et réchauffement climatique, Entreprises et Histoire, $\mathrm{n}^{\circ} 86$, avril 2017.

${ }^{7}$ G. Ripert, Les aspects juridiques du capitalisme moderne, Paris, LGDJ, 1951, p. 42. 
d'opportunités. Elles ont tous les moyens de l'unité dans l'action et n'ont aucune existence juridique en tant qu'organisation. Et la « science» économique dominante ne voit en elles que du marché, un peu plus dense autour de «nœuds de contrats ». La globalisation actuelle, c'est aussi une faillite de la pensée de ce qui nous arrive...

Patrick Verley : Je pense qu'il est possible et même souhaitable de prendre un peu plus de recul chronologique. Une nouvelle conception de l'entreprise naît en Europe entre le XVI et le XVIII ${ }^{\mathrm{e}}$ siècle. Auparavant, une opération de commerce international consistait en le montage d'une société par de nombreux armateurs et négociants pour armer un bateau, acheter des marchandises, les vendre, revenir avec une cargaison de retour, la vendre, liquider l'opération, partager les bénéfices ou les pertes, puis recommencer «l'entreprise». La conception d'une stratégie de développement de long terme qui a été celle des compagnies a impliqué des changements profonds intellectuels et organisationnels. Pour trouver à résoudre le problème du financement des achats dans un contexte de balance commerciale déficitaire, l'idée était non seulement de s'engager dans le commerce intra-asiatique pour faire des profits mais encore de faire de l'arbitrage sur monnaie, en jouant sur les différents rapports de valeur entre l'or et l'argent selon les places. Or une telle activité devait s'inscrire dans la durée pour mettre en relation tous les points commerciaux de la périphérie de l'océan Indien et des mers de Chine, du Moyen-Orient et des marchés européens. Le projet était déjà clair pour le premier gouverneur des Indes néerlandaises, Jan Pieterszoon Coen. Il impliquait une vision à très long terme, la capacité d'investir dans cette stratégie, en un mot une conception de l'entreprise comme organisme se développant dans le très long terme, et perdurant quels que soient les changements de dirigeants. La proximité avec les États a sans aucun doute favorisé cette conception moderne d'une entreprise-organisation, avec une division interne des tâches (l'organigramme de l'East India Company ressemble d'une manière surprenante à celui des entreprises chandlériennes, avec des divisions fonctionnelles et des « cols blancs »).

\section{Peut-on tracer une ligne de continuité entre elles et les entreprises contemporaines ?}

Patrick Verley : En fait, ce type de compagnies ne survit pas à la chute des monarchies absolues (au début du XIX ${ }^{\mathrm{e}}$ siècle, la compagnie anglaise des Indes est un anachronisme sur la défensive). Le commerce international qui forme le réseau porteur de la mondialisation économique et financière repose alors sur des maisons de négoce traditionnelles dans leur organisation alliant opérations sur marchandises, opérations financières, dont la prise en charge d'emprunts d'État et d'opérations d'arbitrage sur monnaies. C'est un milieu fermé avec un réseau de correspondants à l'échelle internationale, qui gravitent autour des gouvernements : les historiens anglais Peter J. Cain et Anthony G. Hopkins ont parlé de gentlemanly capitalism pour leur pays ${ }^{8}$, alliance pour diriger entre l'aristocratie, la haute banque et le négoce international. Enfin, la fin du XIX ${ }^{\mathrm{e}}$ siècle et le début du $\mathrm{XX}^{\mathrm{e}}$ sont reconnus comme une période

\footnotetext{
${ }^{8}$ P. J. Cain and A. G. Hopkins, "Gentlemanly capitalism and British expansion overseas I. The Old colonial system, 1688-1850", Economic History Review, vol. 39, n 4, 1986, p. 501-525; id., "Gentlemanly capitalism and British expansion overseas II: new imperialism, 1850-1945”, Economic History Review, vol. 40, n 1, 1987 , p. 1-26; id., British Imperialism: Innovation and Expansion 1688-2000 [1993], 2e éd. augmentée, Londres, Longman, 2001.
} 
de mondialisation croissante (essentiellement financière et monétaire), mais elle voit une montée des protectionnismes (le XIX ${ }^{\mathrm{e}}$ siècle a été majoritairement protectionniste, mais il y a intensification). Ce n'est pas contradictoire. On voit en même temps le développement des multinationales. La multinationalisation est liée au protectionnisme. Il s'agit de pénétrer les marchés de l'intérieur, pour exemple pour les sociétés américaines, suisses, françaises (Renault et Michelin en Russie, aux États-Unis). Les protectionnismes ont les mêmes résultats dans l'entre-deux guerres (GM rachète Opel et Vauxhall, Ford continue à implanter des usines...). Le transfert technologique est une autre dimension de cette multinationalisation.

Jean-Philippe Robé : En ce qui concerne les pratiques de gestion, un domaine fondamental et appelant toujours des études est celui de la comptabilité. Elle a d'abord été inventée pour permettre au marchand de séparer ses activités commerciales de celles de son foyer, de savoir au terme d'une période où il en est, ce qu'il a, ce qu'il doit et ce qui lui est dû. Puis avec la montée des investissements, la naissance de l'entreprise, la comptabilité va devoir évoluer. Là aussi, l'usage de la société par actions va jouer un rôle majeur : les actionnaires n'étant pas redevables des dettes sociales, les créanciers doivent avoir une image fidèle du patrimoine de leur seul débiteur : la société avec laquelle ils traitent. Puis la comptabilité va se « financiariser » dans le sens où elle va être adaptée pour répondre aux besoins des investisseurs financiers qui veulent pouvoir faire des comparaisons entre entreprises au niveau mondial. Cette comptabilité-là joue un rôle fondamental, car elle pousse à la création de «valeur actionnariale », ce qui ne passe pas nécessairement par la création de vraie valeur, de plus de biens ou services. Un habile montage permettant d'échapper au paiement de 100 millions en impôts crée autant de «valeur actionnariale ». Mais il détruit autant en services publics, en internalisation des externalités négatives et en correction des inégalités. C'est aujourd'hui un des savoirs majeurs de la globalisation et du pilotage des activités des entreprises.

\section{Parmi les entreprises, quels ont été les gagnants et les perdants des mondialisations passées ? Quels sont les gagnants et les perdants de la mondialisation actuelle ? Quels sont leurs profils respectifs ?}

Jean-Philippe Robé : Les perdants sont toujours ceux qui continuent à jouer à un jeu alors que les règles ont changé. Ceux qui se refusent à faire de l'optimisation fiscale, ceux qui n'externalisent pas, ceux qui « jouent le jeu » du système social qui a pu être le nôtre se mettent en position de perdants. Il faut le comprendre pour agir là où c'est possible et ne pas se contenter d'incantations.

Patrick Verley : La mondialisation est un processus qui produit, en effet, des gagnants et des perdants dans une certaine conflictualité. Les XVII ${ }^{\mathrm{e}}$ et $\mathrm{XVIII}{ }^{\mathrm{e}}$ siècles sont des périodes de conflits ouverts où les États mettent leurs armées et leurs marines au service des intérêts industriels. La question est le contrôle du système commercial mondial et du marché des produits industriels. Puis le XVIII ${ }^{\mathrm{e}}$ siècle est jalonné par le conflit franco-britannique... qui se termine en 1815 par la victoire britannique, après que Napoléon a tenté de faire, par la création d'une économie continentale, une reconversion de l'économie atlantique française antérieure. En revanche, de 1815 à 1880 les relations économiques entre les principales puissances 
économiques sont relativement stables et apaisées. Les années 1880 et 1890 voient la montée des tensions qui se manifestent par des escalades douanières.

Cette période est particulièrement intéressante car elle montre que la question des gagnants et des perdants ne connaît pas de réponse définitive. À la fin du XIX ${ }^{\mathrm{e}}$ siècle, le système commercial antérieur est bousculé par l'arrivée de nouveaux producteurs industriels, grands ou petits. On pense toujours à l'Allemagne et ses industries nouvelles (électricité, chimie de synthèse) en oubliant qu'elles pèsent encore peu dans les exportations en 1914 qui sont toujours dominés par des produits plus anciens comme le textile. Mais l'industrialisation est aussi le fait de très nombreux pays dans le monde qui font du textile bon marché en concurrence directe avec les entreprises britanniques, car il s'agit des mêmes qualités : c'est d'ailleurs l'expansion des marchés des constructeurs de machines britanniques qui ont livré des usines clés en main dans le monde entier qui a suscité cette concurrence. La conflictualité est accrue par le ralentissement de la croissance après 1870. Il n'y a plus de place pour une division internationale du travail simple comme auparavant, du fait des nombreux pays producteurs. On voit, par exemple, apparaître dans les années 1880 apparaître en Angleterre dans le patronat cotonnier le thème de la concurrence « déloyale » des entreprises indiennes (en fait en général anglo-indiennes) qui produisent à des prix si bas qu'on ne peut les concurrencer parce qu'elles exploitent, dit-il, leur main-d'œuvre de manière scandaleuse. Ces bonnes âmes obtiennent l'extension d'une loi britannique sur les conditions de travail protégeant la main-d'œuvre en Inde...

\section{Passons maintenant aux principaux impacts positifs et négatifs de chacune de ces mondialisations. Quels en sont les principaux bénéficiaires ou les principales victimes ?}

Jean-Philippe Robé : Nous parlons à l'échelle d'un millénaire... Les victimes sont nombreuses. Ce sont d'abord des milliers de civilisations qui n'ont pas eu les moyens de résister aux diverses formes d'invasion de la civilisation occidentale. Ce sont des milliers de langues, des cultures entières qui sont perdues. L'inventaire serait terrifiant. Puis la plus grande victime, c'est certainement notre environnement naturel. Nous avons exterminé d'innombrables espèces animales et, aujourd'hui, nous sommes en passe de rendre notre planète inhabitable pour notre espèce. Notre civilisation est une forme de virus - mortel et dont nous ne savons pas encore nous soigner.

Évidemment, en face, il faut mettre à notre crédit les formidables avancées dans la science, la médecine, la connaissance, une forme de «progrès » économique, l'accroissement de la durée de vie, etc. La vie est moins « solitary, poor, nasty, brutish, and short » ${ }^{9}$ qu'elle a pu l'être. Le défi de notre temps est de concilier ce que nous savons faire de plus formidable avec une société et une nature préservées. Ce n'est pas impossible; il nous faut juste comprendre notre histoire partagée...

Patrick Verley : Pour faire ce bilan, il me semble intéressant de se pencher sur la prise de conscience d'effets « pervers » de la mondialisation, réels ou non. En fait le patronat a été

\footnotetext{
${ }^{9}$ T. Hobbes, Leviathan, 1651.
} 
pour beaucoup dans la prise de conscience populaire précoce de la mondialisation des marchés de produits. Par exemple Peter Scholliers a montré que dans les années 1840 le patronat textile de Gand expliquait les effets négatifs de la mécanisation sur les ouvriers par la pression de « la concurrence internationale », pour détourner la colère ouvrière vers un bouc émissaire ; la question a fait l'objet de débats parlementaires ${ }^{10}$. Le spectre de la mondialisation est alors agité. Il y a néanmoins un tournant intellectuel entre 1860 et 1880. Il me semble qu'en France par exemple, au début des années 1860 , les patrons considèrent leurs homologues étrangers, anglais en l'occurrence, comme des concurrents, mais sans acrimonie, au même titre que des concurrents d'autres provinces : on voit ainsi des industriels anglais venir déposer à Paris pour l'enquête industrielle, ouvrir leurs livres de comptes, comparer leurs structures de coût avec leurs collègues français, en toute sincérité. Dans l'enquête de 1870, on sent déjà un changement d'attitude. Lors de la dépression des années 1880 en Grande-Bretagne qui affecte beaucoup l'industrie textile, les patrons de Manchester détournent la colère des ouvriers vers les entreprises indiennes. Avec les nationalismes croissants, ce thème allait avoir beaucoup de succès, en particulier dans l'entre-deux-guerres.

\section{Quels sont les types de régulations importants face aux impacts négatifs de l'actuelle mondialisation ?}

Jean-Philippe Robé : Le premier rôle des entreprises est bien sûr de jouer les États les uns contre les autres. Mais il y a aussi des dirigeants d'entreprise qui ont compris que la logique de système dans laquelle nous sommes est mortifère et qui essaient de faire en sorte que des normes viennent limiter les moyens de la concurrence pour que le match de boxe ordonné ne se transforme pas en bataille généralisée sans règles.

Pour donner une réponse plus complète à votre question, il faut partir d'une compréhension précise de la notion de droit de propriété. Le droit de propriété n'est pas un droit qui porte sur les choses : c'est un droit du propriétaire à l'égard de toutes les autres personnes juridiques. Le propriétaire dispose par principe du droit de faire ce qu'il veut de l'objet de sa propriété. Le droit «de régulation » n'apporte que des exceptions à cette compétence de principe. Le droit de propriété est une règle de compétence. Réglementer ne consiste qu'à apporter des dérogations à cette compétence de principe. Le droit de propriété n'est pas affecté dans sa définition : on a juste déplacé le titulaire du pouvoir de décision par rapport à un domaine précis, qui s'impose par dérogation au propriétaire. Le curseur entre autonomie et hétéronomie, propriété et loi, privé et public etc. a juste été déplacé. Mais cette possible évolution de cette frontière est inhérente à la constitution du système libéral. Et déréguler, ce n'est jamais que rendre le pouvoir de réguler à son titulaire initial : le propriétaire. En quoi estce que cela est important? Dès l'origine, il y a dans le droit de propriété cette idée que des questions apparaissant au niveau « macro », que l'on appelle un peu rapidement " politiques », peuvent et même doivent pouvoir se traduire par l'adoption de règles réduisant l'autonomie du

\footnotetext{
${ }^{10} \mathrm{P}$. Scholliers, «Mots et pratiques. L'industrie cotonnière gantoise, les crises et la perception patronale de la concurrence internationale, 1790-1914 », Revue d'histoire du XIX $X^{e}$ siècle, $\mathrm{n}^{\circ}$ 23, $2^{\mathrm{e}}$ semestre 2001, p. 121-142.
} 
propriétaire. La propriété n'est qu'une compétence de principe ; elle appelle, pour fonctionner, remplir pleinement son rôle, la possibilité que soient adoptées des règles qui vont venir internaliser les externalités négatives, corriger les inégalités et taxer les richesses pour fournir les services publics ou les infrastructures nécessaires.

Ce que la globalisation affecte, c'est cette capacité d'adopter des règles, de réguler. Le Système des États n'arrive pas à faire face aux défis auxquels nous sommes confrontés car les curseurs sont structurellement tirés vers l'autonomie, le privé, la propriété. Et nous assistons, pour beaucoup avec un sentiment d'impuissance, à une érosion des services publics, à une baisse de qualité des infrastructures, à une montée des externalités négatives - voyons le réchauffement climatique que l'Accord de Paris de 2015 est incapable d'endiguer - et à un accroissement destructeur des inégalités sociales.

Il nous faut inventer un cadre institutionnel qui accompagne la globalisation. Nous avons besoin des États, mais il ne faut pas trop compter sur eux. Par exemple, le problème du réchauffement climatique est connu depuis 40 ans et très peu a été fait. Il ne faut pas refuser l'obstacle et, là aussi, continuer à jouer à un jeu avec ses anciennes règles alors qu'elles ont changé. Notre problème, depuis toujours, c'est le pouvoir et sa domestication. Nous avons réussi à domestiquer le pouvoir d'État, à le soumettre à des règles, à vivre en État de droit. Il nous faut maintenant œuvrer à faire que les entreprises deviennent des entreprises de droit, que les pouvoirs qui sont les leurs soient reconnus et donc soient soumis à des règles. Ce n'est pas simple mais ce n'est pas impossible. Là encore, à nous de connaître notre histoire et d'en suivre les enseignements.

Nous avons en fait peu de leviers d'action. Ou, plutôt, nous n'avons guère de moyens d'action à grande échelle. Je crois à l'efficacité de «micro-dispositifs » bien placés dans notre Système de Pouvoirs, et j'en ai expliqué quelques-uns. J'ai aussi beaucoup travaillé à la formulation de ce que l'on appelle un peu improprement «l'objet social de l'entreprise » qui verra le jour dans la loi PACTE. Mais au-delà, et de façon urgente, il nous faut enrichir notre comptabilité des organisations productives. Aujourd'hui, tout est mesuré à partir d'une norme de préservation du capital financier. Il augmente, on a fait des gains ; il diminue, on a fait des pertes. Mais on ne prend pas en compte les autres formes de capital que l'activité productive met en jeu, tel le capital environnemental, et singulièrement notre capital de $\mathrm{CO}_{2}$ restant à émettre. Face à l'urgence climatique, il faudrait que dans toute comptabilité organisationnelle figure en face de l'émission de $\mathrm{CO}_{2}$ à émettre le coût de remplacement de ce capital consommé. Il ne s'agit pas de créer un prix artificiel : les dispositifs inventés ne fonctionnent (logiquement) pas. Mais on peut très bien mettre en face d'une consommation de capacité de $\mathrm{CO}_{2}$ le coût d'un puit de carbone équivalent. Et ce sont les acteurs de marché qui doivent le demander ; sinon, ils ne savent pas dans quoi ils investissent et remplissent mal leur rôle vis-à-vis de leurs propres investisseurs.

Je crois que le plus important, c'est de comprendre puis d'expliciter ce qui nous arrive. Nous ne vivons pas un accident de l'histoire. Nous vivons la continuité d'un système de pouvoirs qui a muté, s'est métamorphosé, mais dans lequel il y a des invariants. Ces invariants, ce sont que les individus ont des droits et que nulle organisation, qu'elle soit publique ou privée, ne peut les en priver. Ensuite, c'est que nous avons besoin de prendre des décisions collectives 13 
car nous avons des choses en commun, une res publica. Nous avons développé des institutions territoriales pour ce faire - au premier rang desquelles l'État, qui doit fournir des services publics, internaliser les externalités négatives et corriger les inégalités qui semblent excessives au domus. Le tout est financé par l'impôt décidé par la représentation nationale qui est aussi celle qui fixe le niveau d'internalisation des externalités négatives et de correction des inégalités. L'État est manifestement empêché de remplir ses missions du fait de la globalisation. Il faut donc déplacer le lieu de production des normes collectives et responsabiliser, constitutionnaliser les entreprises. Il faut leur laisser leur autonomie, mais empêcher qu'elle ne devienne destructrice. C'est sur ces bases que tous les penseurs des sciences sociales soucieux de demain doivent se mobiliser. 